\title{
A New Placement Scheme of Distributed Generation in Power Grid*
}

\author{
Zhipeng Jiang', Tiande Guo ${ }^{2}$, Wei Pei ${ }^{3}$ \\ ${ }^{1}$ Academy of Mathematics and Systems Science, Chinese Academy of Sciences, Beijing, China \\ ${ }^{2}$ University of Chinese Academy of Sciences, Beijing, China \\ ${ }^{3}$ Institute of Electrical Engineering, Chinese Academy of Sciences, Beijing, China \\ Email: lstjzp07@yahoo.com.cn
}

Received March, 2013

\begin{abstract}
Smart grid gets more and more popular today. Distributed generation is one of the key technologies, and especially, the integration problem of the distributed generation is an important issue. Especially, the location and capacity of the distributed generation play an important role for the performance of the distribution network. In this paper, an optimization model to minimize the loss cost of the unsatisfied demand is given. This model is based on a reliability computing method which avoiding power flow calculation in a previous work. Then the model is used on the IEEE-123 nodes experiment network and a result of five distributed generation placement is got.
\end{abstract}

Keywords: Smart Grid; Distributed Generation; Optimal Integration; Optimization Model

\section{Introduction}

The smart grid being more and more popular today and distributed generation is one of the key technologies in it. The distributed generation device is an independent generation, small modular and compatible with the environment whose power is from several kilowatts to 50 megawatt. The power of the distributed generation is owned by the power department, users or the third party, which is used to meet the particular demand of the power system and users. For example, adjusts the peak, supplies the power for remote users and residents, decreases the cost of the power transmission and transformation and improves the reliability of the power supply. The distributed generation is deployed near the users in a distributed way and it's a system which can independently provide power. The distributed generation mainly includes the internal combustion engine micro gas turbine, wind power generation as well as photovoltaic cells which use liquid or gas as the fuel. The distributed generation is an important supplement of self-healing strategy, which plays an important role in peak averting, valley filling and load balancing. However, it also brings about new problems. For example, some types of distributed generation are stochastic, and increase the volatility of the system voltage.

There are many issues concerned in distributed gen-

"This paper is supported by the Important Knowledge Innovation Project of Chinese Academy of Sciences No.Y225011EK2 eration problem, among which the optimal integration is one of the important problems. It involves many aspects of factors. It has not only to find a proper optimization objective, but also to analyze various elements like the location, size and type of the distributed generation. Hence, it is a very complicated issue. The location of distributed generation is a location problem in the operations research, and it's an NP (Non-Deterministic Polynomial) problem which needs an appropriate algorithm to solve it. The constraints need to synthesize the influence of distributed generation on power grid when connecting the grid, the equilibrium conditions of power gird and voltage as well as the randomness of distributed generation; while objective function requires comprehensive consideration of operation cost and power consumption.

\section{Related Work}

The In recent years, a variety of methods on the optimal integration of distributed generation have been arisen. A main method is establishing an optimization model with multiple indicators as objective function and some characteristics and restrictions of power grid as constraints. The location and the access capacity of the distributed generation can be achieved by solving the optimization model. The most important object among these indicators is to minimize the line loss [1-3]. In [1], the authors gave a method of line loss minimization based on analytical method. The mathematical programming method is used in [2] and in [3] the genetic algorithm is adopted. The 
cost minimization is also an important objective considered frequently. The representation of the cost is different in different situation. For instance, the authors in [4] consider the minimization of the cost from the perspective of distributed generation developers while the authors in [5] consider it from that of operators, both them adopts traditional mathematical optimization methods. What mentioned above are all single-objective function model and algorithm. For multi-objective programming, in [6] the genetic algorithm based on trust region is given, which optimizes five objectives including minimizing power loss to find the optimal location and size of distributed generation. The randomness of load and distributed generation is considered in [7]. And its objective function is given in the form of probability load current in [8], in which the authors suppose that there is correlation between distributed generations and loads. The method in [9] is based on the Strength Pareto Evolutionary Algorithm (SPEA), and the objective function contains the simple stochastic simulation of the distributed generation and load, but this method is not superior to the weighted single-objective programming, so the authors suggest to adopting both of the two methods to seek optimal solution set. Literature [10] refers to calculate the optimal operation of distributed generation according to the method of multi-objective programming, with considering the uncertainty of distributed generation (e.g. the wind power) and the relevance between the wind power generation and the weather. Besides, in the literature, the authors represent the changing condition of wind power as time changes by the Markov's transition matrix. The randomness of loads is considered when planning the distributed generation in [11].

All the works above provide various objectives and constrains. In most of these works heuristic algorithms are used to solve the optimization models such as Genetic Algorithm, Simulated Anneal Algorithm, and Particle Swarm Optimization Algorithm, etc. A new method of reliability calculation of the power grid without considering the power flow calculation is proposed in [12]. However, the reliability calculation result is much consistent with the actual reliability. Based on this method, in this paper, an optimization model for minimizing the total loss cost caused by the unsatisfied demand, and the model is used on the IEEE-123 nodes experiment network.

\section{An Optimization Model Based on Minimum Total Loss Cost Caused by the Unsatisfied Demand}

\subsection{The Basic Model}

Suppose there is a main power source supplying power in the micro-grid with $N$ load points, and the capacity of the main power can satisfy all the load points' demand. In order to keep the power grid stable, that is, to keep it run normally when the main power source is out of order or the power load increases suddenly at some nodes, the distributed generation is added. Consider there is $M$ distributed power generations will be integrated into the power grid. Suppose each generation can directly connect to only one load nodes and transport the power to other nodes hop by hop.

For the convenience, in this problem, the capacity of each distributed generation and the loads of each point are supposed to be constant and only the active power is considered. Meanwhile, according to the assumption in [12], an important assumption in this model is that when a node receives power from a distributed generation directly or from other nodes, it satisfies its own demand as much as possible then transport the excess power to its neighbor nodes. Only micro grid is studied in this paper, so the transmission loss is not considered in the model. The model is as follows:

$$
\begin{array}{ll}
\min & \sum_{i} k_{i} \cdot P_{i}^{\text {unsatisfy }} \\
\text { s.t. } & P_{i}^{\text {unsatisfy }}=\max \left(P_{i}^{\text {use }}-P_{i}, 0\right) \\
& P_{i}^{\text {surplus }}=\max \left(P_{i}-P_{i}^{\text {use }}, 0\right) \\
& P_{i}=\sum_{j \in N(i)} P_{j i}+\sum_{k} x_{i k} \cdot C_{k} \\
& \sum_{j \in N(i)} P_{i j} \leq P_{i}^{\text {surplus }} \\
& P_{i j} \cdot P_{j i}=0 \\
& X_{i k}=0 \text { or } 1
\end{array}
$$

\subsection{The Explanation of the Variables}

- $\quad P_{i}^{\text {unsatisfy }}$ : The unsatisfied power demand of Node $i$

- $P_{i}^{\text {surplus }}:$ The residual power of Node $i$

- $P_{i}^{\text {use }}$ : The power demand of Node $i$

- $P_{i}$ : The total power that Node $i$ can get

- $\quad P_{i j}$ : The transmission power from Node $i$ to Node $j$

- $C_{k}$ : The capacity of distributed generation $k$

- $\quad k_{i}$ : The weight of Node $i$

- $\quad x_{i k}=\left\{\begin{array}{lc}1 & \text { distributed generation connect with node } \mathrm{i} \\ 0 & \text { else }\end{array}\right.$

\subsection{The Explanation of the Objective Function}

- The objective function $\sum_{i} k_{i} \cdot P_{i}^{\text {unsatisfy }}$ indicates minimizing the total loss cost caused by the unsatisfied demand

\subsection{The Explanation of the Constraints}

- $P_{i}^{\text {unsatisfy }}=\max \left(P_{i}^{\text {use }}-P_{i}, 0\right)$ : If the total power that 
Node $i$ gets is less than the demand of node $i$, the unsatisfied demand of Node $i$ will equal to $P_{i}^{\text {use }}-P_{i}$; Else, it will equal to 0 ;

- $P_{i}^{\text {surplus }}=\max \left(P_{i}-P_{i}^{\text {use }}, 0\right)$ : If the total power that

Node $i$ gets is more than or equal to its demand, the residual energy of Node $i$ will equal to $P_{i}-P_{i}^{\text {use }}$; Else, it will equal to 0 .

- $\quad P_{i}=\sum_{j \in N(i)} P_{j i}+\sum_{k} x_{i k} \cdot C_{k}$ : The total received power of node $i$ is equal to the total energy supplied by the distributed generation directly connected with node $i$ and the power transported from the neighbor nodes of node $i$.

- $\quad \sum_{j \in N(i)} P_{i j} \leq P_{i}^{\text {surplus }}$ : The residual power of the node $i$ can be transported to its neighbor nodes through any way of distribution, or keep it to itself.

- $\quad P_{i j} \cdot P_{j i}=0:$ The power can only be transported in one direction on each line

\section{Solution and Simulation}

In this paper, the standard testing network of IEEE-123 nodes is adopted to carry out the simulation test, the load and weight of the nodes are both constant and the units of the nodes' load and the capacity of the distributed generation are all kilowatt $(\mathrm{kW})$. The network structure is shown in Figure 1, and the parameters of the nodes are shown in Table 1

On the test network of IEEE-123 nodes above, five distributed generations are set, whose capacities are: $C 1$ $=700, C 2=650, C 3=500, C 4=350, C 5=600$ respectively.

We establish a model according to the method proposed in this paper, and solve it by Lingo. The results of the distributed generation's placement and power transmission are as follows. The place result of the distributed generation is shown in Figure 2 and the result of the power transmission (direction and size) between the nodes is shown in Table 2 . The final value of the objective function (that is the total loss cost) is: 6868.2. And

- Distributed generation P1 is placed on the node 36;

- Distributed generation P2 is placed on the node 30;

- Distributed generation P3 is placed on the node 160 ;

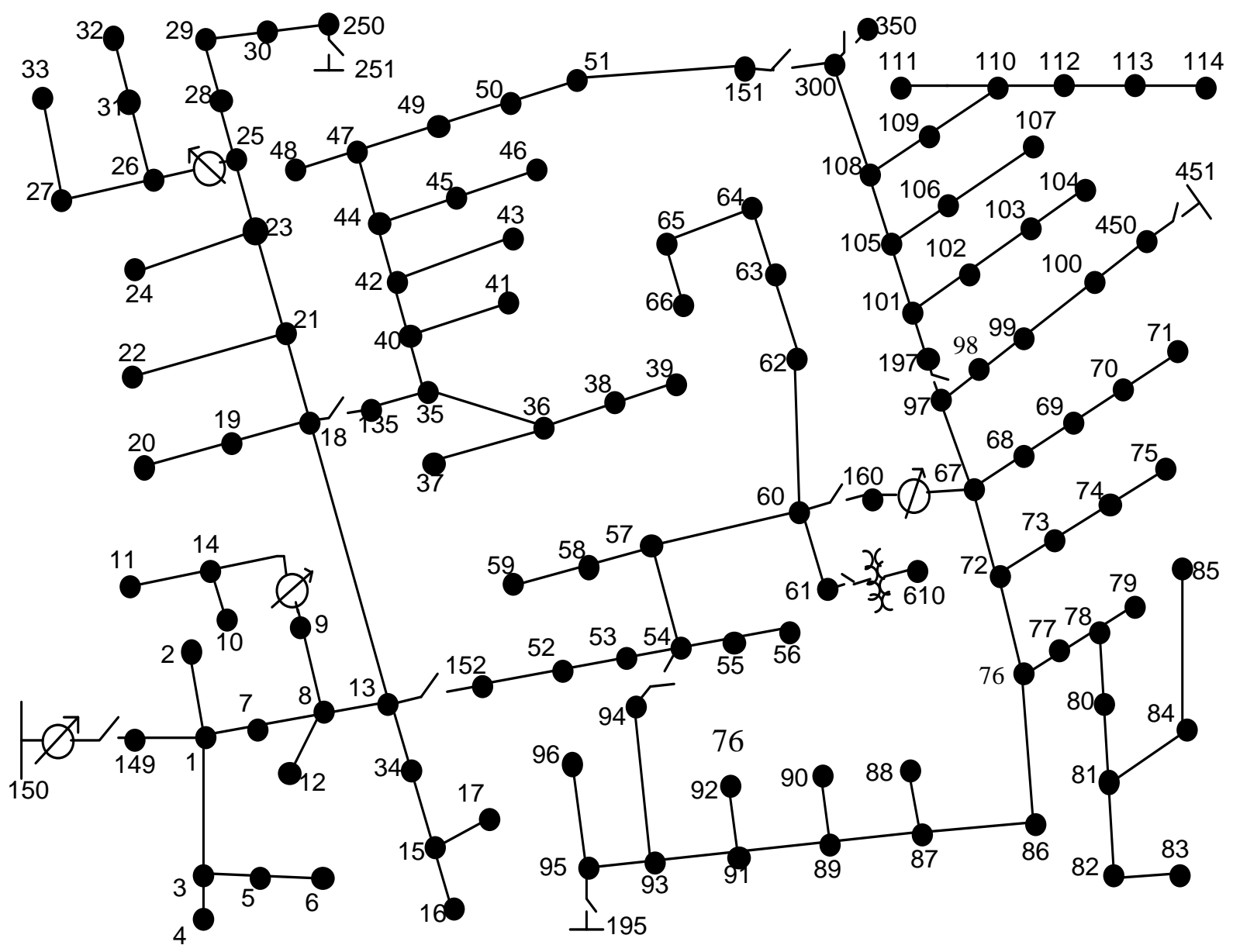

Figure 1. The network structure of the IEEE-123 nodes. 
Table 1. The parameters of the nodes.

\begin{tabular}{|c|c|c|c|c|c|c|c|c|}
\hline id & load & weight & id & load & weight & id & load & weight \\
\hline 1 & 35 & 8.41 & 41 & 20 & 2.77 & 80 & 40 & 9.97 \\
\hline 2 & 40 & 7.25 & 42 & 20 & 3.26 & 81 & 40 & 1.7 \\
\hline 3 & 20 & 3.85 & 43 & 20 & 6.54 & 82 & 35 & 4.98 \\
\hline 7 & 35 & 9.55 & 44 & 40 & 5.26 & 84 & 40 & 1.96 \\
\hline 4 & 20 & 1.31 & 45 & 35 & 4.16 & 83 & 20 & 9.66 \\
\hline 5 & 40 & 4.95 & 47 & 20 & 8.48 & 85 & 20 & 1.04 \\
\hline 6 & 20 & 4.43 & 46 & 35 & 6.27 & 87 & 40 & 7.97 \\
\hline 8 & 40 & 7.89 & 48 & 20 & 5.95 & 88 & 40 & 4.14 \\
\hline 12 & 35 & 8.16 & 49 & 70 & 9.25 & 89 & 40 & 8.36 \\
\hline 9 & 20 & 2.68 & 50 & 70 & 3.57 & 90 & 35 & 8.82 \\
\hline 13 & 40 & 5.41 & 51 & 40 & 7.81 & 91 & 40 & 1.76 \\
\hline 14 & 35 & 5.01 & 52 & 20 & 7.78 & 92 & 35 & 4.6 \\
\hline 34 & 35 & 6.82 & 53 & 40 & 4.42 & 93 & 40 & 3.34 \\
\hline 18 & 40 & 7.38 & 54 & 40 & 6.11 & 94 & 35 & 8.2 \\
\hline 11 & 35 & 7.79 & 55 & 35 & 1.68 & 95 & 40 & 4.88 \\
\hline 10 & 40 & 3.48 & 57 & 20 & 1.49 & 96 & 20 & 9.2 \\
\hline 15 & 20 & 7.12 & 56 & 35 & 5.78 & 98 & 20 & 2.64 \\
\hline 16 & 35 & 6.9 & 58 & 20 & 8.01 & 99 & 40 & 3.37 \\
\hline 17 & 40 & 2.46 & 60 & 20 & 9.41 & 100 & 40 & 2.31 \\
\hline 19 & 20 & 2.07 & 59 & 20 & 2.17 & 450 & 40 & 2.22 \\
\hline 21 & 40 & 5.49 & 61 & 20 & 6.12 & 101 & 35 & 8.82 \\
\hline 20 & 35 & 9.64 & 62 & 35 & 5.22 & 102 & 35 & 6.22 \\
\hline 22 & 40 & 4.06 & 63 & 40 & 1.11 & 105 & 20 & 5.95 \\
\hline 23 & 40 & 6.27 & 64 & 40 & 4.03 & 103 & 35 & 2.3 \\
\hline 24 & 35 & 3.01 & 65 & 75 & 2.46 & 104 & 40 & 8.68 \\
\hline 25 & 40 & 7.76 & 66 & 70 & 8.15 & 106 & 40 & 6.6 \\
\hline 26 & 35 & 3.3 & 67 & 75 & 3.8 & 108 & 40 & 4.16 \\
\hline 28 & 35 & 5.55 & 68 & 35 & 5.76 & 107 & 35 & 5.62 \\
\hline 27 & 40 & 7.29 & 72 & 20 & 2.49 & 109 & 40 & 4.62 \\
\hline 31 & 35 & 9.02 & 97 & 35 & 6.42 & 300 & 40 & 1.68 \\
\hline 33 & 20 & 9.63 & 69 & 35 & 3.37 & 110 & 35 & 3.16 \\
\hline 29 & 40 & 5.92 & 70 & 40 & 6.89 & 111 & 35 & 2.11 \\
\hline 30 & 40 & 2.25 & 71 & 20 & 7.2 & 112 & 20 & 2.66 \\
\hline 250 & 40 & 2.34 & 73 & 40 & 7.73 & 113 & 20 & 3.16 \\
\hline 32 & 35 & 3.32 & 76 & 40 & 5.05 & 114 & 40 & 4.76 \\
\hline 35 & 20 & 8.57 & 74 & 105 & 1.75 & 135 & 20 & 1.45 \\
\hline 36 & 40 & 3.29 & 75 & 40 & 3.06 & 149 & 35 & 9.12 \\
\hline 40 & 35 & 8.33 & 77 & 40 & 9.22 & 152 & 35 & 9.5 \\
\hline 37 & 35 & 3.19 & 86 & 40 & 2.37 & 160 & 35 & 5.42 \\
\hline 38 & 40 & 9.36 & 78 & 20 & 8.43 & 197 & 35 & 5.4 \\
\hline 39 & 20 & 4.15 & 79 & 35 & 5.85 & & & \\
\hline
\end{tabular}




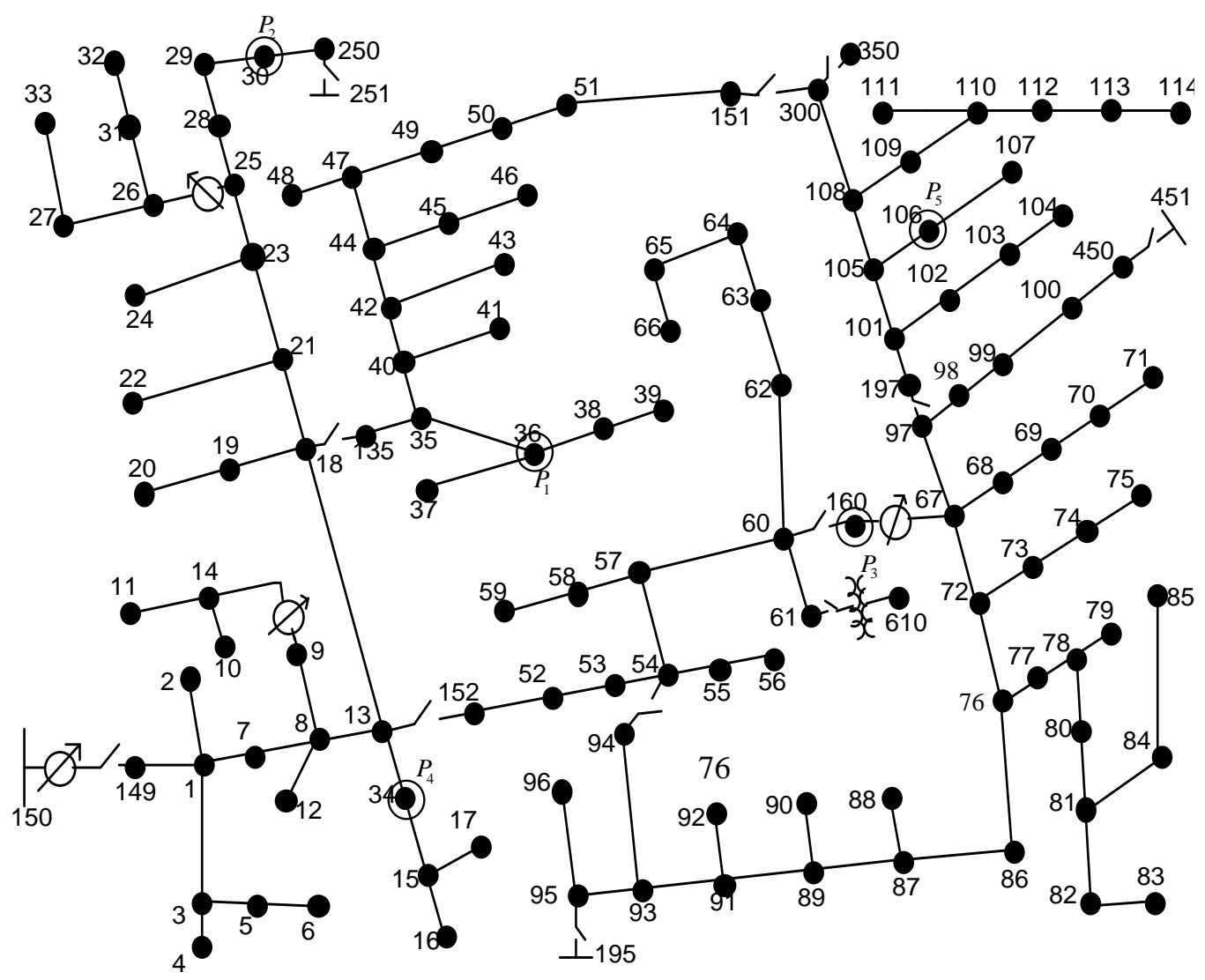

Figure 2. The placement result of the distributed generation.

Table 2. The power flow transmission result.

\begin{tabular}{|c|c|c|c|c|c|}
\hline $1->2$ & 40 & $29->28$ & 570 & $72->76$ & 295 \\
\hline $1->3$ & 80 & $30->29$ & 610 & $76->77$ & 100 \\
\hline $1->149$ & 35 & $34->13$ & 260 & $76->86$ & 155 \\
\hline $3->5$ & 60 & $34->15$ & 55 & $77->78$ & 60 \\
\hline $5->6$ & 20 & $35->40$ & 525 & $78->80$ & 40 \\
\hline $7->1$ & 190 & $35->135$ & 20 & $86->87$ & 115 \\
\hline $8->7$ & 225 & $36->35$ & 565 & $87->89$ & 75 \\
\hline $8->12$ & 35 & $36->37$ & 35 & $89->90$ & 35 \\
\hline $8->9$ & 90 & $36->38$ & 60 & $101->102$ & 110 \\
\hline $9->14$ & 70 & $38->39$ & 20 & $101->197$ & 35 \\
\hline $13->8$ & 390 & $40->41$ & 20 & $102->103$ & 75 \\
\hline $14->11$ & 35 & $40->42$ & 470 & $103->104$ & 40 \\
\hline $15->16$ & 35 & $42->43$ & 20 & $105->101$ & 180 \\
\hline $18->13$ & 170 & $42->44$ & 430 & $105->108$ & 325 \\
\hline $18->19$ & 55 & $44->45$ & 70 & $106->105$ & 525 \\
\hline $19->20$ & 35 & $44->47$ & 320 & $106->107$ & 35 \\
\hline $21->18$ & 265 & $45->46$ & 35 & $108->109$ & 245 \\
\hline $21->22$ & 20 & $47->48$ & 20 & $108->300$ & 40 \\
\hline $23->21$ & 325 & $47->49$ & 280 & $109->110$ & 205 \\
\hline $25->23$ & 365 & $49->50$ & 210 & $110->111$ & 35 \\
\hline $25->26$ & 130 & $50->51$ & 40 & $110->112$ & 135 \\
\hline $26->27$ & 60 & $67->72$ & 355 & $112->113$ & 115 \\
\hline $26->31$ & 35 & $67->97$ & 35 & $113->114$ & 40 \\
\hline $27->33$ & 20 & $72->73$ & 40 & $160->67$ & 465 \\
\hline $28->25$ & 535 & & & & \\
\hline & & & & & \\
\hline
\end{tabular}

- Distributed generation P4 is placed on the node 34;

- Distributed generation P5 is placed on the node 106 ;

\section{Conclusion}

The optimal integration problem of distributed generation is one of the key issues of the smart power grids. In this paper we propose an optimization model of placing the distributed generation, aiming to minimize the total loss cost caused by the unsatisfied power of every node. Meanwhile, we use this model to a five distributed power generation placement problem on the network of IEEE-123 nodes. The result of the distributed generation placement and the power transmission is got.

\section{REFERENCES}

[1] C. Wang and M. Hashem, “Analytical Approaches for Optimal Placement of Distributed Generation Sources in Power Systems," IEEE Transactions on Power Systems, 2004, 19.

[2] S. Narayan, S. M. Rau and W. M., "Yih-heu Optimum Location of Resources in Distribution Planning," IEEE Transactions on Power Systems, 1994, Vol. 9.

[3] J. O. Kim, S. W. Nam, S. K. Park and C. Singh, "Dis- 
persed Generation Planning Using Improved Hereford Ranch Algorithm," Electric Power Systems Research, 1998, Vol. 47, pp. 47-55. doi:10.1016/S0378-7796(98)00038-8

[4] H. L. Willis and W. G. "Scott Distributed Power Generation,” Planning and Evaluation. New York, USA: Marcel Dekker; 2000, ISBN: 0-8247-0336-7.

[5] R. C. Dugan, T. E. McDermott and G. J. Ball, "Planning for Distributed Generation,” IEEE Industry Applications Magazine, 2001.doi:10.1109/2943.911193

[6] G. Celli, E. Ghiani, S. Mocci and F. Pilo, “A Multi-objective Formulation for the Optimal Sizing and Siting of Embedded Generation in Distribution Networks," In: Power tech conference proceedings, 2003 IEEE, Vol. 1, 2003.doi:10.1109/PTC.2003.1304113

[7] G. Celli, E. Ghiani, S. Mocci and F. Pilo, “A Multi-objective Evolutionary Algorithm for the Sizing and Siting of Distributed Generation, IEEE Transactions on Power System, 2005, 20.

[8] G. Celli, S. Mocci, F. Pilo and R. Cicoria, "Probabilistic
Optimization of MV Distribution Networks in Presence of Distributed Generation. In: Proceedings of the 14th PSCC; 2002.

[9] E. Haesen, J. Driesen and R. Belmans, “A Long-term Multi-objective Planning Tool for Distributed Energy Resources. In: IEEE PES Power Systems Conference \& Exposition, 2006, p. 741-747.

[10] A. C. Rueda-Medina, J. M. Lopez-Lezama and A. Padilha-Feltrin, A Multi-objective Programming Approach for the Optimal Operation of Distributed Generation Considering Uncertainty.

[11] A. G. Marinopoulos, M. C. Alexiadis and P. S. Dokopoulos, "Energy Losses in a Distribution Line with Distributed Generation Based on Stochastic Power Flow," Electric Power Systems Research, Vol. 81, 2011, pp. 1986-1994.doi:10.1016/j.epsr.2011.06.006

[12] I.-S. Bae and J.-O. Kim, "Reliability Evaluation of Distributed Generation Based on Operation Mode," IEEE Tranactions Power Systems, Vol. 22, 2007, pp. 785-790. doi:10.1109/TPWRS.2007.894842 